\title{
Fluorine F 18 FEQA
}

National Cancer Institute

\section{Source}

National Cancer Institute. Fluorine F18 FEQA. NCI Thesaurus. Code C66936.

The anilinoquinazoline derivative FEQA radiolabeled with the positron-emitting

radioisotope fluorine $F 18$ with epidermal growth factor receptor (EGFR) binding and

radioisotopic activities. Fluorine $F 18$ FEQA irreversibly binds to EGFR, allowing imaging of EGFR-positive tumor cells with positron emission tomography (PET). 